\title{
Mapeamento da interceptação de chuva na bacia hidrográfica do Alto Juruá, Acre
}

\author{
Rainfall interception mapping in the Alto Juruá hydrographic basin, Acre \\ Mapeo de interceptación de lluvia en la cuenca hidrográfica del Alto Juruá, Acre
}

Recebido: 04/12/2021 | Revisado: 10/12/2021 | Aceito: 17/12/2021 | Publicado: 02/01/2022

\author{
Cassia Fernanda de Souza Farias \\ ORCID: https://orcid.org/0000-0002-6882-4351 \\ Universidade Federal do Acre, Brasil \\ E-mail: cassia.farias@ sou.ufac.br \\ Jefferson Vieira José \\ ORCID: https://orcid.org/0000-0003-1384-0888 \\ Universidade Federal do Acre, Brasil \\ E-mail: jefferson.jose@ufac.br \\ Kelly Nascimento Leite \\ ORCID: https://orcid.org/0000-0003-1919-9745 \\ Universidade Federal do Acre, Brasil \\ E-mail: kellyleite14@hotmail.com \\ Moisés Damasceno Souza \\ ORCID: https://orcid.org/0000-0002-7019-6497 \\ Universidade Federal do Acre, Brasil \\ E-mail: moisesdamasceno789@gmail.com \\ Marcos Antônio Correa Matos Amaral \\ ORCID: https://orcid.org/0000-0002-7623-6445 \\ Universidade Federal do Oeste do Pará, Brasil \\ E-mail: marcos.esalq@ hotmail.com \\ Sonaira Souza da Silva \\ ORCID: https://orcid.org/0000-0003-2177-4577 \\ Universidade Federal do Acre, Brasil \\ E-mail: sonaira.silva@ufac.br
}

\begin{abstract}
Resumo
As perdas por interceptação das águas pluviais são frequentemente negligenciadas devido às dificuldades de medição e grande variabilidade espacial e temporal. As perdas podem ser significativas e, portanto, ter um impacto severo no balanço hídrico de uma bacia hidrográfica. O presente trabalho teve como objetivo obter a interceptação pluvial na bacia hidrográfica do Alto Juruá (BHAJ), por meio do modelo de Gash, com base em dados de sensoriamento remoto. A plataforma para o processamento dos dados foi o Google Earth Engine, no qual permitiu avaliar e comparar as variáveis de precipitação pluvial, índice de vegetação com diferença normalizada e índice de área foliar (IAF), em escala mensal e anual, no período de 2003 a 2016. Dessa forma, foi possível observar que a interceptação possui uma forte relação com IAF e a cobertura vegetal, registrando uma média anual de 11,2\% de interceptação de chuva pela floresta dentro da BHAJ, apresentando suas maiores porcentagens nos períodos mais chuvosos.
\end{abstract}

Palavras-chave: Área Foliar; Amazônia; Modelo de Gash.

\begin{abstract}
Rainwater interception losses are often neglected due to measurement difficulties and great spatial and temporal variability. Losses can be significant and therefore have a severe impact on the water balance of a watershed. The present work had as objective to obtain the pluvial interception in the hydrographic basin of the Alto Juruá (BHAJ), through the Gash model, based on remote sensing data. The platform for data processing was the Google Earth Engine, which allowed the assessment and comparison of rainfall variables, normalized difference vegetation index and leaf area index (LAI), on a monthly and annual scale, in the period of 2003 to 2016. Thus, it was possible to observe that the interception has a strong relationship with LAI and vegetation cover, recording an annual average of $11.2 \%$ of rain intercept by the forest within the BHAJ, with its highest percentages in the rainiest periods.
\end{abstract}

Keywords: Foliar Area; Amazon; Gash Model.

\section{Resumen}

Las pérdidas por interceptación de agua de lluvia a menudo se pasan por alto, debido a las dificultades de medición y la gran variabilidad espacial y temporal. Las pérdidas pueden ser significativas y, por lo tanto, tener un impacto severo en el balance hídrico de una cuenca. El presente trabajo tuvo como objetivo obtener la interceptación pluvial en la cuenca hidrográfica del Alto Juruá (BHAJ), a través del modelo Gash, con base en datos de teledetección. La plataforma para el procesamiento de datos fue Google Earth Engine, que permitió la evaluación y comparación de las 
variables de precipitación, índice de vegetación de diferencia normalizada e índice de área foliar (LAI), en una escala mensual y anual, en el período de 2003 a 2016. Así, se pudo observar que la interceptación tiene una fuerte relación con la LAI y la cobertura vegetal, registrando un promedio anual de 11,2\% de interceptación de lluvia por el bosque dentro del BHAJ, con sus mayores porcentajes en los períodos más lluviosos.

Palabras clave: Área Foliar; Amazonia; Modelo de Gash.

\section{Introdução}

A floresta Amazônica tem um papel importante na chuva local e regional contribuindo para o ciclo hidrológico e transporte de umidade dentro e fora do território brasileiro, afetando o ciclo hidrológico e os níveis dos rios amazônicos (Marengo \& Souza Jr., 2018). A bacia hidrográfica da Amazônia é considerada a maior presente no planeta (Silva et al., 2010), dito isso todas os processos hídricos ocorrentes na mesma são responsáveis por um grande volume de água, tanto em relação a entrada e saída da região, um dos processos mais influentes e que geram uma grande saída hídrica é a evapotranspiração. A evapotranspiração é um suprimento que contribui para formação de grandes massas umidades que se movimentam em várias orientações que chegam até os estados do Centro-sul do Brasil (Marengo, 2018).

Tendo em vistas todas as mudanças climáticas locais e certos distúrbios ocorrentes nas florestas da zona de convergência tropical sul, o que gera problemas de abastecimento para a agricultura e reservatórios urbanos com estiagens e períodos chuvosos, só dão indícios da influência desse processo na floresta. Portanto, pode-se dizer que modificações nos padrões de evapotranspiração podem afetar os padrões de chuva em diversas regiões do planeta (O'connor et al., 2019).

A chuva que cai sobre uma bacia hidrográfica florestal é naturalmente fracionada em três parcelas: parte dela é interceptada e armazenada pela vegetação, e evapora durante ou após o evento de chuva; outra parte cai livremente sobre o solo, sem interferência da vegetação, ou depois de ser interceptada e gotejar; e a terceira parte alcança o solo escoando pelos troncos, após ser interceptada (Giglio \& Kobiyama, 2013).

A interceptação da chuva pela vegetação é a retenção de parte da precipitação acima da superfície do solo (Brasil et al., 2017). O volume retido, temporariamente, é perdido por evaporação, retornando à atmosfera. (Freitas et al., 2016; Xiao \& Mcpherson, 2016; Alves et al., 2018). Consequentemente, a interceptação da chuva é um fator importante em modelos hidrológicos que simulam processos do balanço hídrico, como evaporação, transpiração e escoamento superficial (Jong e Jetten, 2007), sendo responsável por 7 a 22\% da chuva total em florestas tropicais (Giglio \& Kobiyama, 2013).

A interceptação vegetal depende de vários fatores: características da chuva e condições climáticas, chuvas anteriores, tipo e densidade da vegetação e período do ano. As características principais da chuva são intensidade e volume precipitado (Balbinot, 2008; López, 2016). Com base nesses fatores os modelos hidrológicos têm grande importância na previsão de enchentes e, portanto, são uma ferramenta valiosa para os governantes no planejamento e adequação de medidas visando essas previsões.

Os estudos de monitoramento de interceptação são poucos e concentrados na Mata Atlântica ou, quando decorrentes de iniciativas internacionais, na Amazônia (Giglio \& Kobiyama, 2013). A quantificação da interceptação tem diferentes abordagens disponíveis, como as medidas diretas, com dispositivos instalados em parcelas no campo. Devido às dificuldades de medição, medidas indiretas, por meio do balanço hídrico, são mais comuns (Lima, 2008; Sá et al., 2015).

Os modelos de medidas indiretas mais aplicados para estimar a perda por interceptação da floresta são o Gash e Rutter (Sá et al., 2015). No modelo de Gash as variáveis de evaporação, chuva, capacidade de armazenamento de vegetação, índice de área foliar (IAF) podem ser obtidos por sensoriamento remoto, obtendo a perda por interceptação em cada pixel de imagem de satélite (Cui \& Jia, 2014). Diante do exposto o presente estudo objetivou estimar a interceptação pluvial na bacia hidrográfica do Alto Juruá (BHAJ), com base em dados de sensoriamento remoto. 


\section{Metodologia}

\section{Área de estudo}

A bacia hidrográfica do Alto Juruá (BHAJ) está localizada no Norte do Brasil, entre os estados do Acre e Amazonas e a República do Peru. A cobertura vegetal natural da área é classificada como floresta Amazônica, de vegetação latifoliada equatorial, ou seja, formada por palmeiras, cipós, árvores de grande porte e folhas largas (Gligio \& Kobiyama, 2013). A altimetria da bacia varia de $156 \mathrm{~m}$ a $354 \mathrm{~m}$. Na Figura 1a, pode-se observar a área de estudo com a sua rede de drenagem e os seus níveis de elevação altímetro e, Figura 1b a classe de vegetação na bacia hidrográfica do Alto Juruá. Conforme apresentado na Figura 1b, a BHAJ tem as maiores ocorrências das classes de vegetação do tipo Vegetação Ombrófila Aberta de Terras Baixas, Floresta Ombrófila Densa Terras Baixas, Vegetação Secundária e Atividades Agrárias, Vegetação Ombrófila Aberta Aluvial e Floresta Ombrófila Densa Submontana.

Figura 1. Mapa de localização (a) e classe de vegetação (b) na bacia hidrográfica do Alto Juruá.

a)

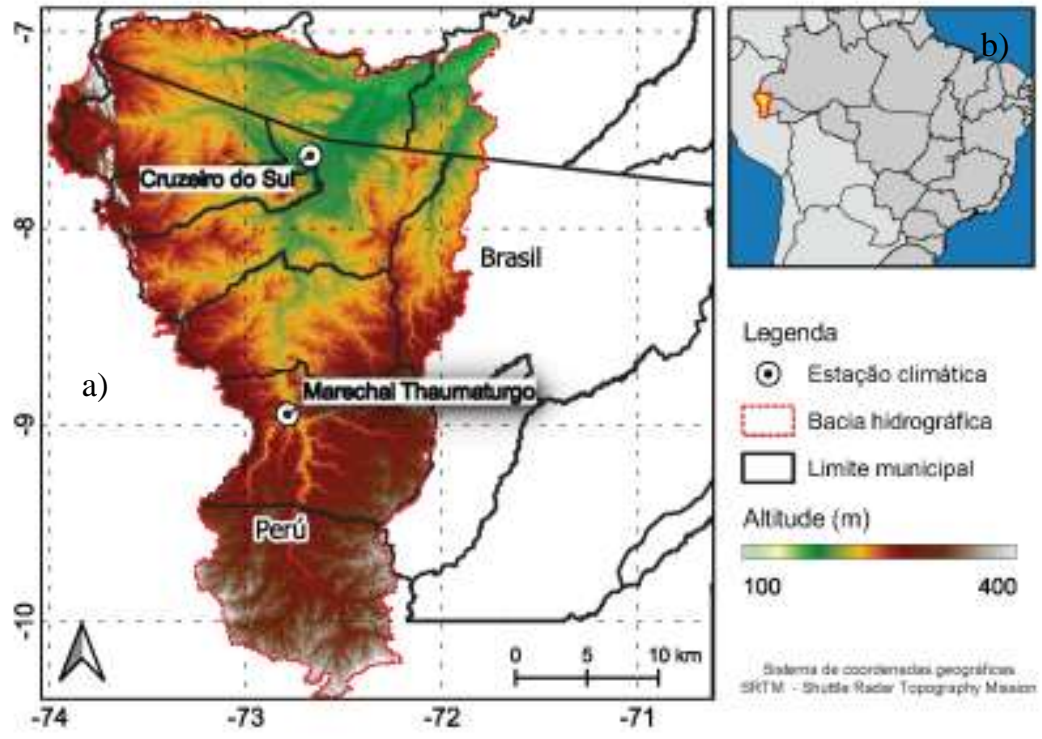

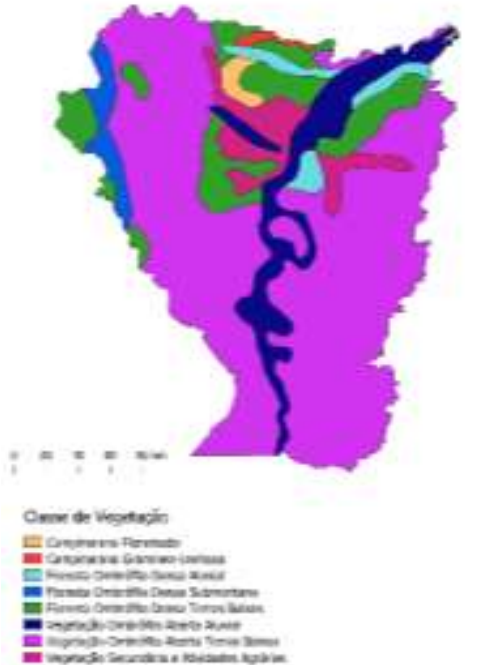

Fonte: Autores.

O tipo de clima da região é considerado Af, caracterizado por clima tropical equatorial, sem estação seca (Alvares et al., 2014). A evapotranspiração média anual da região é de 1728 mm (José et al., 2020), com temperatura do ar média anual de $25^{\circ} \mathrm{C}$ e chuva média anual de $2227 \mathrm{~mm}$. O período úmido acontece entre os meses de outubro a abril concentrando $79,4 \%$ da chuva anual e o período menos úmido ocorre entre maio a setembro (Araújo et al., 2020).

\section{Processamento de dados}

A plataforma selecionada para o processamento dos dados e das informações obtidas através de satélites, foi a do ambiente Google Earth Engine (GEE). Este sistema de análise geoespacial é baseado na nuvem, com vários petabyte de informação, obtida por sensoriamento remoto, com capacidade global, empregando a linguagem de programação JavaScript na criação dos algoritmos para tratamento das imagens de satélites (Kumar e Mutanga, 2018).

Sendo assim, por meio da ferramenta Code Editor: https://code.earthengine.google.com/, foi possível programar e executar uma rotina a qual permitisse avaliar as variáveis: precipitação pluvial, altitude, uso e cobertura vegetal, Índice de Área Foliar -IAF e Índice de Vegetação da Diferença Normalizada - NDVI, todas descritas na Tabela 1. Para extrair os valores de interceptação (I), precipitação pluvial (P), índice de área foliar (IAF), cobertura fracionada de vegetação (cf) e índice de 
vegetação da diferença normalizada (NDVI). Com o uso do modelo de Gash foi determinada a interceptação de acordo com a Equação 1.

$$
I=a \times I A F \times\left(1-\frac{1}{1+\frac{1+c f \times \frac{P}{r}}{a \times I A F}}\right)
$$

Em que:

I - Interceptação, \%;

a - Parâmetro empírico, adotou-se $1 \mathrm{~mm}$;

IAF - Índice de área foliar;

cf - Cobertura fracionada de vegetação;

$\mathrm{P}$ - Precipitação pluvial mensal, mm;

r - Número mensal de dias chuvosos.

Tabela 1. Conjuntos de dados usados neste estudo.

\begin{tabular}{|c|c|c|c|c|c|}
\hline Dados & $\begin{array}{c}\text { Resolução } \\
\text { Espacial }\end{array}$ & $\begin{array}{l}\text { Resolução } \\
\text { Temporal }\end{array}$ & $\begin{array}{l}\text { Formato de } \\
\text { dados }\end{array}$ & $\begin{array}{l}\text { Cobertura } \\
\text { temporal }\end{array}$ & Uso \\
\hline MCD15A3H & $500 \mathrm{~m}$ & 4 dias & GeoTiff & 2003-2016 & Dados de índice de área foliar (IAF) \\
\hline MYD13Q1.005 & $250 \mathrm{~m}$ & 16 dias & GeoTiff & 2003-2016 & Dados de NDVI \\
\hline CHIRPS & $500 \mathrm{~m}$ & diário & GeoTiff & 2003-2016 & Dados de precipitação pluvial \\
\hline INMET & - & diário & CSV & $\begin{array}{l}1975-1990 \\
1991-2012\end{array}$ & $\begin{array}{l}\text { Dados de número médio de dias } \\
\text { precipitação pluvial mensal }\end{array}$ \\
\hline SRTM & $30 \mathrm{~m}$ & - & GeoTiff & 2009 & Dados de Altitude \\
\hline RADAMBRASIL & $1: 250.000$ & - & Shapefile & 2019 & Dados de vegetação \\
\hline Hidrográfica Ottocodificada & $1: 250.000$ & - & Shapefile & 2020 & Limite da bacia hidrográfica \\
\hline Limite administrativo & $1: 250.000$ & - & Shapefile & 2020 & Limite estadual \\
\hline
\end{tabular}

SRTM - Shuttle Radar Topography Mission; CHIRPS - Clima Hazards Group InfraRed Precipitation with Station data. Fonte: Autores.

Os índices de vegetação IAF e o NDVI foram obtidos de dois produtos dos sensores MODIS dos satélites Terra e Aqua. O IAF foi obtido do produto MCD15A3H, composto de um conjunto de dados de resolução temporal de 4 dias com tamanho de pixel de 500 metros. O NDVI foi extraído do produto MYD13Q1.005 com 16 dias de resolução temporal e tamanho de pixel de $250 \mathrm{~m}$. Foram utilizados dois tipos de dados de precipitação pluvial.

Os dados de campo de duas estações climáticas localizadas no estado do Acre, sendo elas: Cruzeiro do Sul, Marechal Thaumaturgo mostrado na Tabela 2, foram utilizados para calcular o número mensal médio de dias com chuva (r). Os dados de precipitação pluvial (P) foi obtido via satélite, com o produto CHIRPS/PENTAD, é um conjunto de dados com resolução temporal de 10 dias e resolução espacial de $0,05^{\circ}$. 
Tabela 2. Número médio mensal de dias com chuva nas estações climáticas da bacia hidrográfica do Alto Juruá, Acre, Brasil.

\begin{tabular}{|c|c|c|c|c|c|c|c|c|c|c|c|c|c|c|}
\hline \multirow{2}{*}{ Est. } & \multirow{2}{*}{ Lat. } & \multirow{2}{*}{ Long. } & \multicolumn{12}{|c|}{ Meses } \\
\hline & & & $\mathrm{J}$ & $\mathrm{F}$ & $\mathrm{M}$ & A & $\mathrm{M}$ & $\mathrm{J}$ & $\mathrm{J}$ & $\mathrm{A}$ & $\mathrm{S}$ & $\mathrm{O}$ & $\mathrm{N}$ & $\mathrm{D}$ \\
\hline CZS & $-7,61^{\circ}$ & $-72,68^{\circ}$ & 26 & 25 & 26 & 22 & 21 & 18 & 13 & 14 & 18 & 20 & 22 & 23 \\
\hline MRT & $-8,95^{\circ}$ & $-72,79^{\circ}$ & 26 & 23 & 24 & 19 & 17 & 15 & 8 & 11 & 12 & 18 & 23 & 25 \\
\hline & Média & & 26 & 24 & 25 & 20,5 & 19 & 16,5 & 10,5 & 12,5 & 15 & 19 & 21 & 24 \\
\hline
\end{tabular}

Fonte: INMET - Instituto Nacional de Meteorologia; Est. - Estações; Lat. Latitude; Long. Longitude. CZS - Cruzeiro do Sul (1975-1990); MRT - Marechal Thaumaturgo (1991-2002).

A cobertura fracionada de vegetação (cf) foi calculada a partir do índice de vegetação por diferenças normalizadas (NDVI), onde NDVImax e NDVImin são os valores limites de cobertura vegetal total e solo nu, respectivamente, Equação 2.

$$
C f=1-((N D V I \max -N D V I) /(N D V I \min -N D V I))
$$

\section{Análise dos dados}

Os dados de interceptação pluvial, precipitação pluvial, NDVI e IAF foram apresentados em mapas de distribuição espacial anual no qual foram gerados gráficos de valores médios da BHAJ em escala mensal e anual. Índices estatísticos foram usados para comparar a interceptação e as variáveis precipitação pluvial total, NDVI e IAF na escala mensal e anual na BHAJ. Os índices utilizados foram o coeficiente de determinação $\left(\mathrm{R}^{2}\right)$, correlação de Pearson (R), a raiz do erro quadrático médio (RMSE), o erro médio absoluto (MBE), coeficientes lineares (interceptação) e angulares (inclinação) das equações de regressão.

A partir dos dados obtidos seu processamento ocorreu utilizando o software livre R Statistical ${ }^{\circledR}$ (R Core Team, 2021) e os seus pacotes para obtenção e análise dos dados. Os testes estatísticos de comparação entre a altitude e as variáveis climáticas foi por meio do pacote hydroGOF (Zambrano-Bigiarini, 2020). Os gráficos foram realizados com pacotes ggplot2 (Wickham, 2016) e gridExtra (Auguie, 2017). A ferramenta QGIS 3.18, Sistema de Informação Geografia livre e de código aberto, foi utilizada para o processamento final dos rasters gerados na plataforma GEE na elaboração de mapas.

\section{Resultados e Discussão}

Na Figura 2 são apresentados os resultados das lâminas médias e percentuais referentes a interceptação e precipitação pluvial mensal e anual na bacia hidrográfica do Alto Juruá. A média de precipitação pluvial mensal é de 179 mm, onde observa-se uma diminuição a partir do mês de março, sendo junho o mês menos chuvoso (41,52 mm), com aumento no mês de setembro, (Figura 2a). Os valores anuais de precipitação pluvial apresentam média de 1933,82 mm, e os anos de 2003 a 2005 , 2010, 2014 e 2015 os valores ultrapassam a média e nos anos de 2008 e 2013 foram abaixo da média, 1839,2 e 1848,6 mm, respectivamente (Figura 2b). As maiores precipitações concentram-se na região Norte e Nordeste da BHAJ, tendo volumes de precipitações acima dos 2357,1 mm, já as menores lâminas de chuva se distribuem de forma aleatória pela região (Figura 6). 
Figura 2. Interceptação e precipitação pluvial média (2003-2016) mensal (a) e anual (b) na bacia hidrográfica do Alto Juruá. A linha continua representa a média, enquanto a linha pontilhada a média móvel.
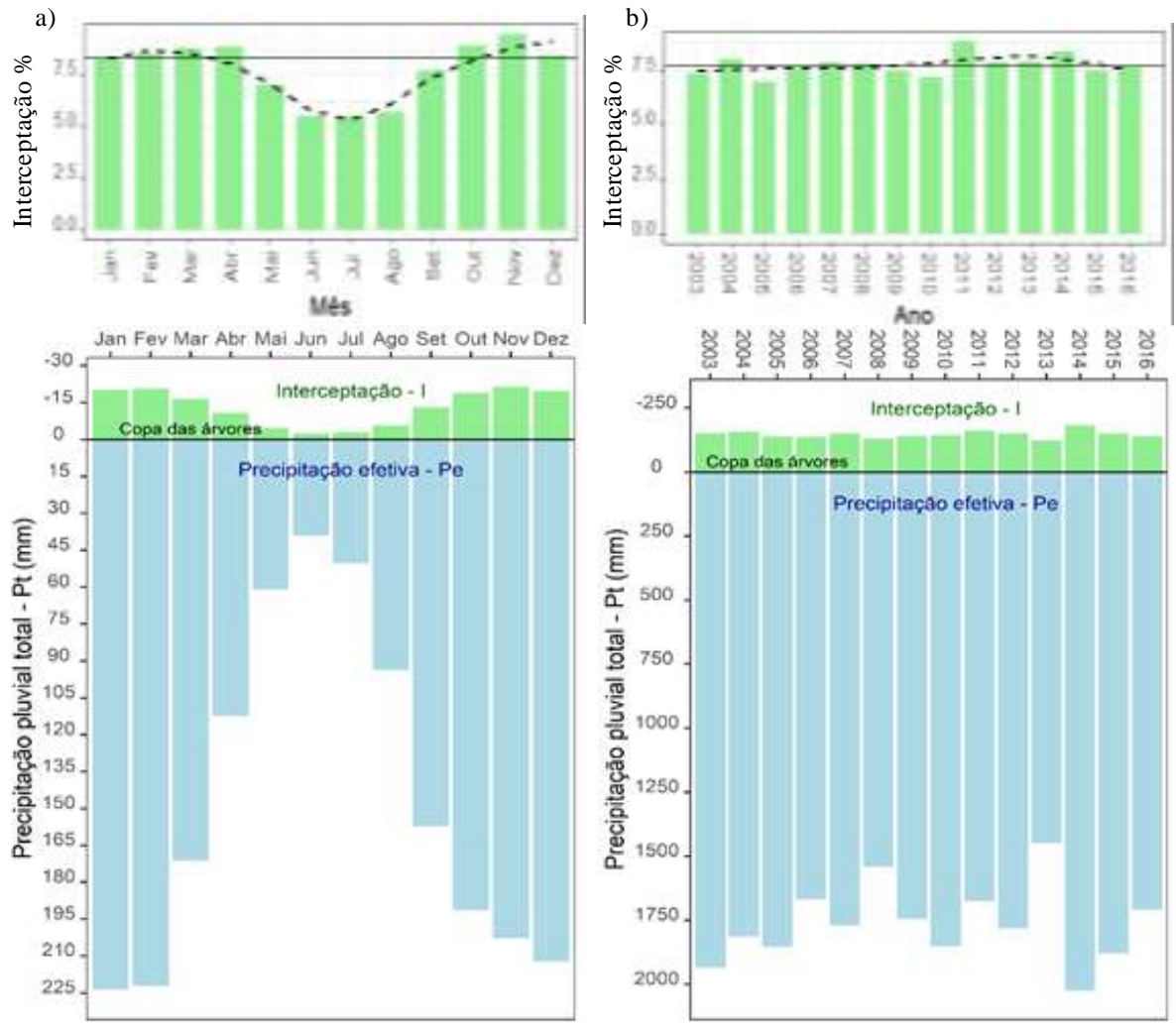

Fonte: Autores.

A capacidade de interceptação pluvial apresentou uma média mensal de 7,6\%, registrando maiores volumes interceptados nos meses que apresentam mais eventos de precipitação evidenciando sua maior porcentagem no mês de novembro com 9,3\% e a menor nos meses de junho, julho e agosto (Figura 2a). Verifica-se as perdas por interceptação entre dois períodos (chuvoso e menos chuvoso). Esta variabilidade é devida às características distintas das precipitações locais que ocorrem nos dois períodos do ano. As diferenças na interceptação entre períodos de precipitação foram verificadas também por Arcova et al. (2003).

Percebe-se que o nível de interceptação ao longo dos anos tem pequenas variações, porém o maior registro de interceptação (8,7\%) não foi obtido no ano que apresentou maior volume de precipitação (2014), (Figura 2b) Sabe-se que as variações interanuais da interceptação podem ser influenciadas por vários fatores como, intensidade e volume da precipitação e quantidade de eventos chuvosos (López, 2016), como observado em estudo desenvolvido em floresta de terra firme na Amazônia Central (Cuartas et al., 2007). Assim como ocorrência de fenômenos adversos, Sousa e Costa (2020) verificaram que a distribuição de chuvas nessa região é resultado da atuação da Zona de Convergência Intertropical (ZCIT), e da formação de Linhas de Instabilidade (LI), sofrendo forte influência nos anos que ocorre eventos de El Niño e La Niña. 
Figura 3. Índice de área foliar (IAF) média anual (2003 a 2016) na bacia hidrográfica do Alto Juruá.

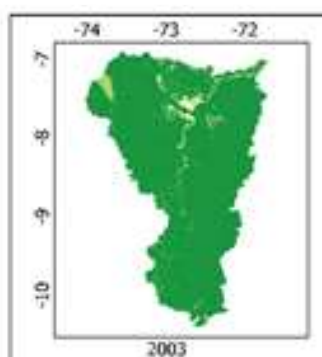

2003

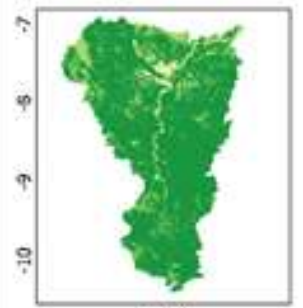

2006
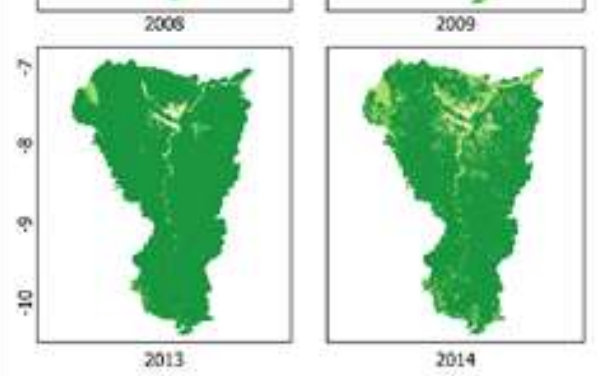

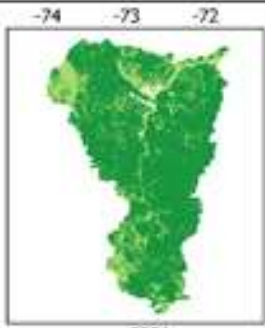

2004
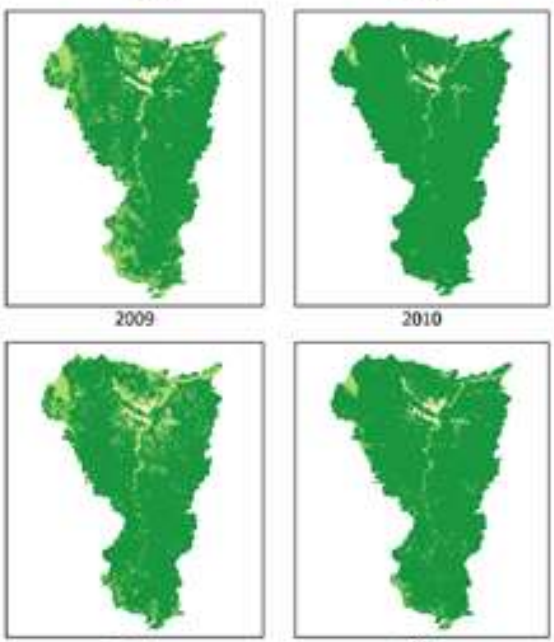

2015

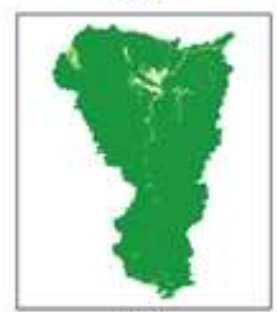

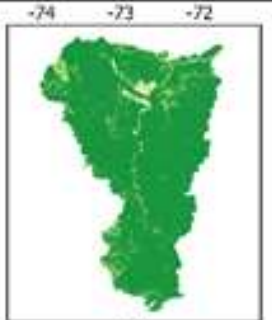

2006

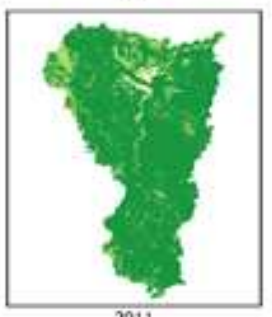

2011

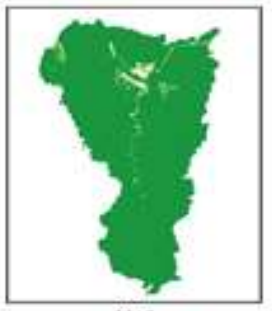

Ind de Area Foliar - LAt

U $<0,20$

$0,20-1,40$

$1,40-2,60$

$2,60-3,80$

미 $>3,80$

Fonte: Autores.

Os dados de interceptação e precipitação total mensal e anual foram submetidos à análise de regressão linear, cujos resultados estão na Figura 4. A interceptação, apresentou forte correlação com a quantidade da precipitação total no período mensal ( $\mathrm{R}^{2}$ de 0,98, RMSE de 159,60), porém relativamente forte no período anual, com $\mathrm{R}^{2}$ igual a 0,62 e RMSE de 1770,91, com tendência de maiores valores de interceptação nos acumulados mais chuvoso.

Figura 4. Diagrama de dispersão de interceptação com a precipitação pluvial em escala mensal (a) e anual (b) na bacia hidrográfica do Alto Juruá. Coeficientes de determinação $\left(\mathrm{R}^{2}\right)$. Correlação de Pearson (R). Erro médio absoluto (MBE). Raiz do erro quadrático médio (RMSE).
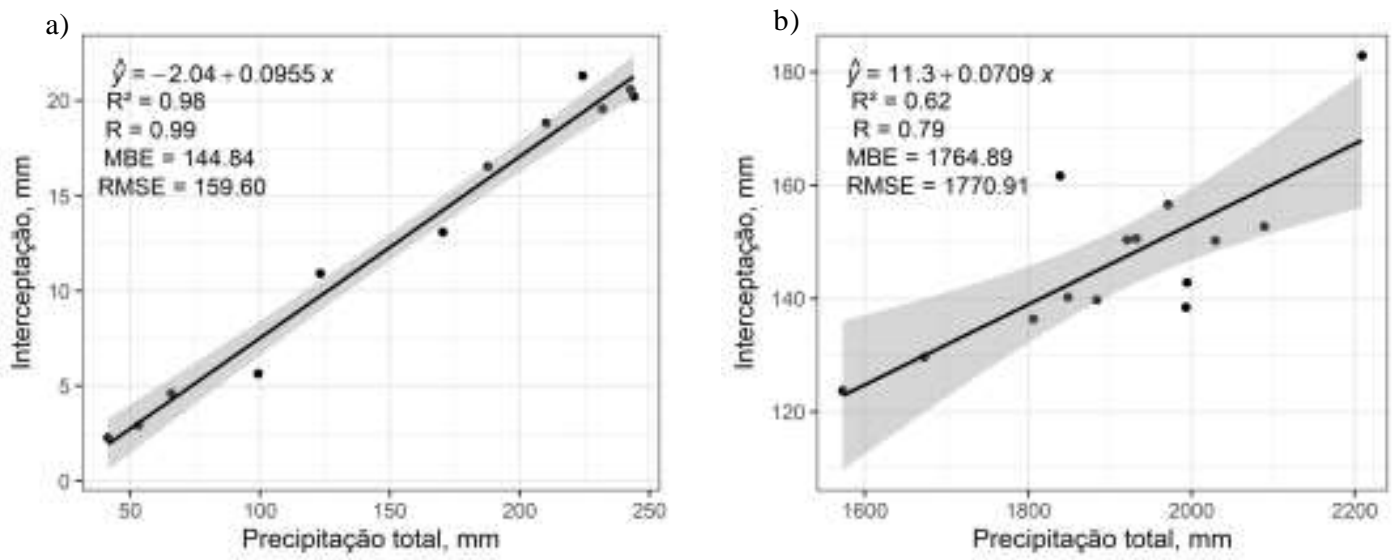

Fonte: Autores. 
A variação espacial da perda de interceptação (Figura 5) é claramente atribuída à variação do uso e cobertura do solo (Figura 1b) e IAF (Figura 3) na BHAJ, além da variação espacial da precipitação pluvial total (Figura 6). A interceptação anual da chuva pela vegetação mostrou que o processo é heterogêneo dentro da mesma área de estudo na BHAJ. Essa variabilidade está em acordo com a suposição de Horton (1919) de que a interceptação depende das características da chuva, como volume, intensidade e frequência (condições meteorológicas) e da vegetação.

As maiores interceptações pluviais concentram-se na floresta da BHAJ, tendo valores de 11,2\%, já os menores níveis de interceptações concentram-se em áreas urbanas (5,8\%) (Figura 5). Cuartas et al. (2007) apresentou uma interceptação média variando de 13 a 23\%, podendo, ainda, variar de 13 a 21\% na estação úmida, e na estação seca, de 12 a $25 \%$. Na pesquisa realizada por Tobón et al. (2000) em Peña Roja - Colômbia, a interceptação variou de 12 a 17\% no período de um ano, ficando dentro do percentual médio de interceptação de regiões de floresta amazônica.

Figura 5. Interceptação média anual (2003 a 2016) na bacia hidrográfica do Alto Juruá.

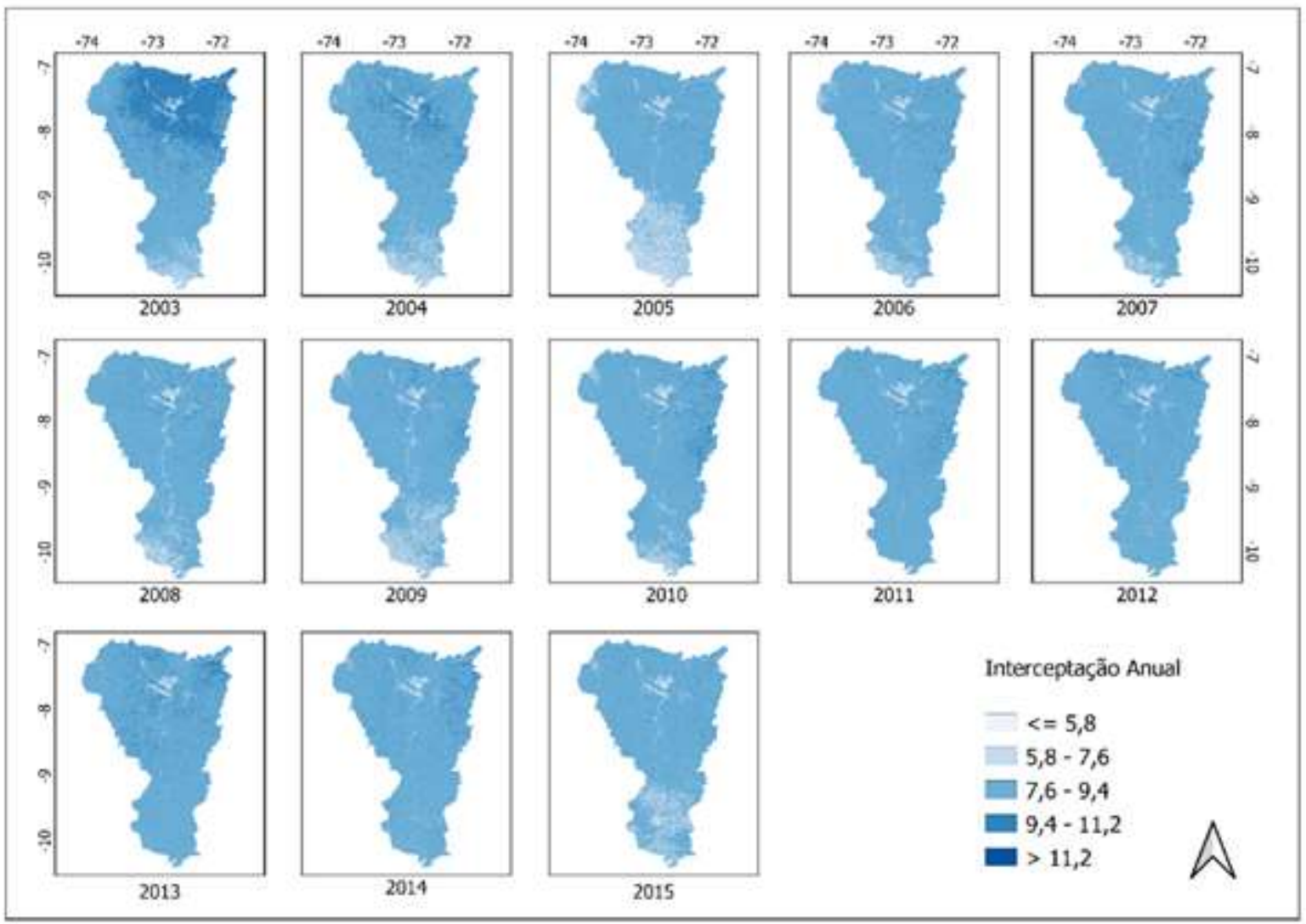

Fonte: Autores.

Conforme apresentado na Tabela 3, no ano de 2003 a interceptação pluvial média pela vegetação foi de 9,45\%, as classes de vegetação do tipo Floresta Ombrófila Densa Aluvial, Campinarana Gramíneo-lenhosa e Campinarana Florestada foram as que apresentaram maiores porcentagens de interceptação, com 9,88, 9,74 e 9,79 \%, respectivamente, e a classe de vegetação do tipo Floresta Ombrófila Densa Submontana obteve a menor porcentagem, com 8,79\%. Pode-se notar que com o passar dos anos esses percentuais têm uma significativa redução, e no ano de 2015 a influência da vegetação na interceptação pluvial reduziu para uma média de $8,42 \%$. 
Tabela 3. Porcentagem da interceptação pluvial anual das classes de vegetação na bacia hidrográfica do Alto Juruá.

\begin{tabular}{|c|c|c|c|c|c|c|c|c|}
\hline \multirow{2}{*}{ Ano } & \multicolumn{8}{|c|}{ Classe de vegetação } \\
\hline & A & $\mathrm{B}$ & $\mathrm{C}$ & $\mathrm{D}$ & $\mathrm{E}$ & $\mathrm{F}$ & G & $\mathrm{H}$ \\
\hline 2003 & $9,79 \pm 0,3$ & $9,74 \pm 0,4$ & $9,88 \pm 0,4$ & $8,79 \pm 0,5$ & $9,46 \pm 0,4$ & $9,32 \pm 0,9$ & $9,17 \pm 0,5$ & $9,45 \pm 0,9$ \\
\hline 2004 & $9,17 \pm 0,4$ & $8,83 \pm 0,4$ & $9,22 \pm 0,4$ & $8,44 \pm 0,5$ & $8,97 \pm 0,3$ & $8,86 \pm 0,9$ & $8,94 \pm 0,4$ & $8,94 \pm 0,9$ \\
\hline 2005 & $8,71 \pm 0,3$ & $8,48 \pm 0,4$ & $8,81 \pm 0,4$ & $7,93 \pm 0,4$ & $8,46 \pm 0,4$ & $8,31 \pm 0,8$ & $8,34 \pm 0,4$ & $8,48 \pm 0,9$ \\
\hline 2006 & $8,51 \pm 0,4$ & $8,67 \pm 0,4$ & $8,83 \pm 0,4$ & $8,19 \pm 0,4$ & $8,6 \pm 0,4$ & $8,36 \pm 0,8$ & $8,54 \pm 0,4$ & $8,55 \pm 0,8$ \\
\hline 2007 & $8,29 \pm 0,4$ & $8,65 \pm 0,4$ & $8,93 \pm 0,4$ & $8,39 \pm 0,4$ & $8,64 \pm 0,4$ & $8,28 \pm 0,8$ & $8,64 \pm 0,1$ & $8,47 \pm 0,9$ \\
\hline 2008 & $8,59 \pm 0,4$ & $8,84 \pm 0,4$ & $8,93 \pm 0,4$ & $8,45 \pm 0,4$ & $8,73 \pm 0,4$ & $8,34 \pm 0,9$ & $8,49 \pm 0,5$ & $8,52 \pm 0,9$ \\
\hline 2009 & $8,68 \pm 0,4$ & $8,97 \pm 0,4$ & $8,97 \pm 0,4$ & $8,14 \pm 0,4$ & $8,76 \pm 0,4$ & $8,32 \pm 0,9$ & $8,35 \pm 0,5$ & $8,47 \pm 0,9$ \\
\hline 2010 & $8,29 \pm 0,3$ & $8,72 \pm 0,4$ & $8,95 \pm 0,4$ & $7,99 \pm 0,3$ & $8,53 \pm 0,4$ & $8,19 \pm 0,8$ & $8,48 \pm 0,4$ & $8,41 \pm 0,8$ \\
\hline 2011 & $8,57 \pm 0,3$ & $8,75 \pm 0,3$ & $8,96 \pm 0,4$ & $8,37 \pm 0,3$ & $8,68 \pm 0,4$ & $8,36 \pm 0,8$ & $8,64 \pm 0,4$ & $8,44 \pm 0,9$ \\
\hline 2012 & $8,96 \pm 0,3$ & $9,05 \pm 0,3$ & $9,05 \pm 0,4$ & $8,39 \pm 0,4$ & $8,83 \pm 0,4$ & $8,54 \pm 0,8$ & $8,57 \pm 0,4$ & $8,49 \pm 0,9$ \\
\hline 2013 & $9,09 \pm 0,4$ & $9,15 \pm 0,3$ & $9,16 \pm 0,4$ & $8,54 \pm 0,4$ & $9,06 \pm 0,3$ & $8,73 \pm 0,8$ & $9,24 \pm 0,4$ & $8,75 \pm 0,9$ \\
\hline 2014 & $8,85 \pm 0,4$ & $8,98 \pm 0,3$ & $9,01 \pm 0,4$ & $8,36 \pm 0,4$ & $8,87 \pm 0,4$ & $8,43 \pm 0,8$ & $8,62 \pm 0,4$ & $8,54 \pm 0,9$ \\
\hline 2015 & $8,48 \pm 0,4$ & $8,65 \pm 0,4$ & $8,67 \pm 0,4$ & $8,15 \pm 0,4$ & $8,51 \pm 0,4$ & $8,17 \pm 0,8$ & $8,28 \pm 0,5$ & $8,26 \pm 0,9$ \\
\hline
\end{tabular}

A - Campinarana Florestada; B - Campinarana Gramineo-Lenhosa; C - Floresta Ombrófila Densa Aluvial; D - Floresta Ombrófila Densa Submontana; E - Floresta Ombrófila Densa Terras Baixas; F - Vegetação Ombrófila Aberta Aluvial; G - Vegetação Ombrófila Aberta Terras Baixas; H - Vegetação Secundária e Atividades Agrárias. Fonte: Autores.

O índice de área foliar (IAF) é a relação entre a área das folhas e todas as folhas da vegetação de uma região e a área projetada no solo. A média anual de IAF foi de 3,86 $\mathrm{m}^{2}$, obtendo seu valor máximo no ano de $2016\left(4,00 \mathrm{~m}^{2} \mathrm{~m}^{-2}\right) .0 \mathrm{valor}$ de IAF igual a 4, por exemplo, significa que cada $\mathrm{m}^{2}$ de área de solo está coberto por uma vegetação em que a soma das áreas das folhas individuais é de $4 \mathrm{~m}^{2}$. De Jong e Jetten (2007) e Oerlemans e Vink (2010) verificaram relações com a interceptação e o IAF para diferentes espécies de vegetação, ou seja, com o aumento dos índices vegetativos, maior é capacidade de interceptação da chuva pelo dossel (Dijk e Bruijnzeel, 2001).

Porém, quando os índices vegetativos são reduzidos, com desmatamento, a capacidade de interceptação da chuva pelo dossel também é reduzida. A vegetação é responsável por segurar (interceptar) as gotas de água da chuva e reduzir a sua velocidade até a chegar ao solo, no entanto, se não houver vegetação a chuva não será interceptada, podendo aumentar o escoamento superficial, afetar os processos de infiltração, e assim, aumentar a vazão dos rios. Desse modo, os eventos de enchentes em períodos de chuvas intensas acabam se potencializando, afetando as áreas urbanas inundando ruas e casas, gerando vários problemas ambientais e econômicos, e em períodos menos chuvosos pode provocar secas severas dos rios. 
Figura 6. Precipitação pluvial média anual (2003 a 2016) na bacia hidrográfica do Alto Juruá.

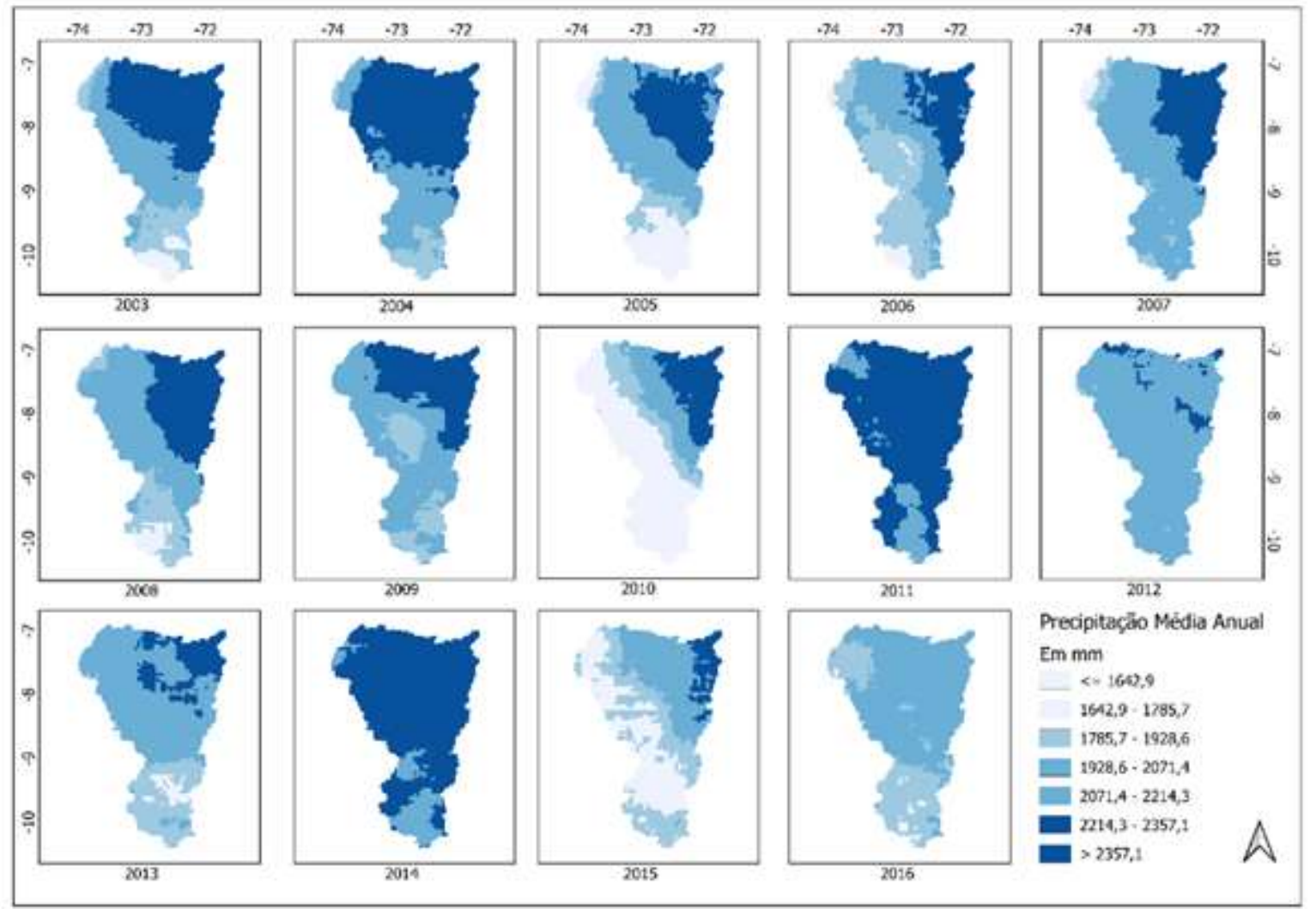

Fonte: Autores.

\section{Conclusão}

Considerando as condições em que o experimento foi conduzido e os resultados obtidos, foi possível chegar às seguintes conclusões na bacia hidrográfica do Alto Juruá:

- A interceptação anual da chuva pela floresta teve média de $11,2 \%$, ou seja, $217 \mathrm{~mm}$ ou $2170 \mathrm{~m}^{3}$ ha $^{-1}$ foi retornando à atmosfera na forma de vapor.

- A substituição da cobertura florestal por áreas urbanizadas aumenta em $1048 \mathrm{~m}^{3} \mathrm{ha}^{-1}$ a água da chuva anual que chega ao solo da bacia hidrográfica.

- As porcentagens de interceptação foram maiores no período chuvoso.

- Entre os anos 2003 e 2016 houve uma redução na interceptação vegetal.

- A interceptação possui uma forte relação com IAF e a cobertura vegetal.

- Foi possível criar mapas de interceptação na bacia hidrográfica do Alto Juruá com base em índices de vegetação por meio de imagens de sensoriamento remoto.

\section{Referências}

Alvares, C. A., Stape, J. L., SentelhaS, P. C., Gonçalves, J. L. de M., \& Sparovek, G. Koppen's Climate Classification Map For Brazil. Meteorologische Zeitschrift, 22(6), 711-728, 2014.

Alves, P. L., Formiga, K. T. M., \& Traldi, M. A. B. (2018). Interferência de Espécies Arbóreas na Interceptação das Águas Pluviais Urbanas. RBCIAMB, (47), 89- 100 .

Araujo, E. S. de, Almeida, M. P. de, Leite, K, N., Silva, J. R. dos S., Araújo, E. A. de, \& Sousa, G. G. de. (2020). Climatic Characterization and Temporal Analysis of Rainfall in the Municipality of Cruzeiro do Sul - AC, Brazil. Revista Brasileira de Meteorologia, 35(4), 577 - 584. 
Arcova, F. C. S., Cicco, V. de, Rocha, P. A. B. (2003). Precipitação Efetiva E Interceptação Das Chuvas Por Floresta De Mata Atlântica Em Uma Microbacia Experimental Em Cunha - São Paulo. R. Árvore, Viçosa-MG, 27(2),.257-262.

Auguie, B. gridExtra: Miscellaneous Functions for "Grid" Graphics, 2017. https://cran.r-project.org/web/packages/gridExtra/gridExtra.pdf.

Balbinot, R., Oliveira, N. K. de., Vanzetto, S. C., Pedroso, K., \& Valerio, A. F. (2008). O papel da floresta no ciclo hidrológico em bacias hidrográficas. Ambiência. 4(1), 131-149

Brasil, J. B., Palácio, H. A. de Q., Araújo Neto, J. R. de, Ribeiro Filho, J. C., Andrade, E. M. de. (2017). Características das Chuvas e Interceptação Vegetal no Bioma Caatinga. Irriga. 22(3), 560-574.

Cuartas, L. A., Tomasella, J., Nobre, A. D., \& Hodnett, M. G. (2007). Interception Water-Partitioning Dynamics for a Pristine Rainforest in Central Amazonia: Marked Differences Between Normal and Dry Years. Agricultural and Forest Meteorology. 145, 69-83.

Cui, Y., \& Jia, L. (2014). A Modified Gash Model for Estimating Rainfall Interception Loss of Forest Using Remote Sensing Observations at Regional Scale. Water. 6, p. 993-1012.

Dijk, A.I.J.M., Bruijnzeel, L.A. (2001). Modelling rainfall interception by vegetation of variable density using an adapted analytical model. Part 2 - Model validation for a tropical upland mixed cropping system. Journal of Hydrology, Amsterdam, 247, 239-62

Freitas, J. P. O., Dias, H. C. T., Silva, E., \&Tonello, K. C. (2018). Precipitação Líquida em Fragmento Florestal Semidecíduo na Cidade de Viçosa, MG. Revista Árvore. 40(5), 793-801.

Giglio, J. N., \& Kobiyama, M. (2013). Interceptação da Chuva: Uma Revisão com Ênfase no Monitoramento em Florestas Brasileiras. Revista Brasileira de Recursos Hídricos. 18(2), 297- 317.

Horton, R. E. (2019). Rainfall Interception. Monthly Weather Review, 26(653), 25-27.

Jong, S. M., \& Jetten, V. G. (2007). Estimating spatial patterns of rainfall interception from remotely sensed vegetation indices and spectral mixture analysis. International Journal of Geographical Information Science. 21(5), 529-545.

José, J. V., Pereira, L. B., Leite, H. M. F., Santos, L. da C., Barros, T. H. da S., leite, K. N. (2020). Métodos empíricos de estimativa da evapotranspiração em clima tropical equatorial. Research Society and Development, 9, e4099108563.

Kumar, L., \& Mutanga, O. (2018). Google Earth Engine Applications Since Inception: Usage, Trends, and Potential. Remote Sens. 10, 2-15.

Lima, W. de P. (2008). Hidrologia Florestal Aplicada ao Manejo de Bacias Hidrográficas. Universidade de São Paulo- USP

López, J. F. (2016). Interceptação de Águas Pluviais em um Fragmento de Mata Atlântica da Reserva Biológica de Duas Bocas, Cariacica (ES) Brasil. Tese (Mestrado em Geografia) Universidade Federal Do Espírito Santo Centro De Ciências Humanas e Naturais Programa De Pós-Graduação Em Geografia Da UFES, Vitoria, p. 143.

Marengo, J. A., \& Souza jr, C. (2018). Mudanças Climáticas: impactos e cenários para a Amazônia. O que o ISP pode fazer por...? São Paulo. p. 1-33.

O'connor, J. et al. The influence of water table depth on evapotranspiration in the Amazon arc of deforestation. Hydrology and Earth System Sciences Discussions, p. 1-22, 2019.

Oerlemans, R.R.R., \& Vink, R. P. (2010). Rainfall Interception Experiments and Interception mapping using Remote Sensing. (Tese de Mestrado) Faculty of Geosciences Department of Physical Geography Utrecht University, p. 108.

R Core Team. R: A language and environment for statistical computing. R Foundation for Statistical Computing, Vienna, 2021. https://www.R-project.org>.

Sá, J. H., Chaffe, P. L., \& Oliveira, D. Y. (2015). Análise comparativa dos modelos de Gash e de Rutter para a estimativa da interceptação por Floresta Ombrófila Mista. Revista Brasileira de Recursos Hídricos, 20(4), 1008- 1018.

Silva D. et al. (2005). Interações entre nuvens, chuvas e a biosfera na Amazônia. Acta Amazônica, 2(35), $215-222$.

Sousa, J. W., \& Costa, D. B. Ajuste de distribuições de probabilidades à série 1970-2010 de precipitação máxima anual de Rio Branco, Acre. Revista Scientia Naturalis, 2(1), 177-187.

Tobón, C., Bouten, W., Sevink, J. (2000). Gross rainfall and its partitioning into throughfall, stemflow and evaporation of intercepted water in four Forest ecosystems in western Amazonia. J. Hydrol. 37, 40-57

Wickham, H. (2016). ggplot2: Elegant Graphics for Data Analysis. Springer-Verlag New York.

Xião, Q., \& Mcpherson, E. G. (2016). Surface water storage capacity of twenty tree species in Davis, California. Journal of Environmental Quality, 45(1), $188-198$.

Zambrano-Bigiarini, M. hydroGOF: Goodness-of-Fit Functions for Comparison of Simulated and Observed Hydrological Time Series, 2020. https://cran.rproject.org/web/packages/hydroGOF/hydroGOF.pdf 